\title{
Uplink Load Estimation in WCDMA
}

Erik Geijer Lundin, Fredrik Gunnarsson, Fredrik Gustafsson

Division of Communication Systems

Department of Electrical Engineering Linköpings universitet, SE-581 83 Linköping, Sweden

WWW: http://WwW. control.isy.liu.se

E-mail: geijer@isy.liu.se, fred@isy.liu.se

fredrik@isy.liu.se

25-mar-02

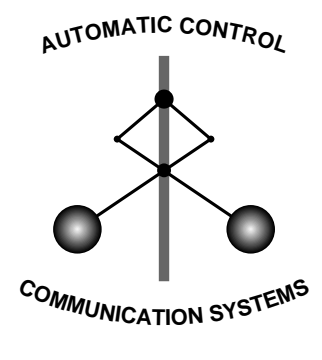

LINKÖPING

Report no.: LiTH-ISY-R-2458

Submitted to RVK'02

Technical reports from the Control \& Communication group in Linköping are available at http://www.control.isy.liu.se/publications. 


\begin{abstract}
All cellular radio systems depend on well performing radio resource management algorithms to efficiently utilize available resources. These algorithms make their decisions based on some sort of resource quantity. In the uplink of a WCDMA system, the total received power at the base stations is a natural choice of such a quantity. Unfortunately this quantity cannot be measured with enough accuracy to be used by resource management algorithms. One way of getting around this problem is to estimate a closely related quantity, namely the uplink noise rise. In this paper, we propose and evaluate four different methods to estimate the noise rise. The estimates are insensitive to users' bit rate and use data readily available in the system. The best performing method estimates, on average, the uplink noise rise with an error of less than $1 \mathrm{~dB}$ for practical load scenarios.
\end{abstract}

Keywords: WCDMA, resource management, uplink load 


\title{
Uplink Load Estimation in WCDMA
}

\author{
Erik Geijer Lundin ${ }^{1}$, Fredrik Gunnarsson ${ }^{2}$ and Fredrik Gustafsson ${ }^{3}$ \\ ${ }^{1}$ Linköpings universitet, 58183 LINKÖPING, SWEDEN. E-mail: geijer@isy.liu.se \\ ${ }^{2}$ Linköpings universitet, 58183 LINKÖPING, SWEDEN. E-mail: fred@isy.liu.se \\ ${ }^{3}$ Linköpings universitet, 58183 LINKÖPING, SWEDEN. E-mail: fredrik@isy.liu.se
}

\begin{abstract}
All cellular radio systems depend on well performing radio resource management algorithms to efficiently utilize available resources. These algorithms make their decisions based on some sort of resource quantity. In the uplink of a WCDMA system, the total received power at the base stations is a natural choice of such a quantity. Unfortunately this quantity cannot be measured with enough accuracy to be used by resource management algorithms. One way of getting around this problem is to estimate a closely related quantity, namely the uplink noise rise. In this paper, we propose and evaluate four different methods to estimate the noise rise. The estimates are insensitive to users' bit rate and use data readily available in the system. The best performing method estimates, on average, the uplink noise rise with an error of less than $1 \mathrm{~dB}$ for practical load scenarios.
\end{abstract}

\section{Introduction}

Crucial for obtaining good utilization of available radio resources are well operating radio resource management algorithms. Such algorithms rely on knowledge of some sort of resource quantity. Examples of such quantities are the number of active users in a cell, which is an example of capacity measures sometimes referred to as hard capacity [1], and total received uplink power which is an example of a soft capacity measure. The main difference is that the latter considers the effects of the radio environment and of multiple cells. Soft capacity is used for uplink admission control in $[1,2,3,4]$. In this paper we will partially focus on the uplink noise rise, i.e., total received interference power, $I$ over background noise power, $N$. Another quantity, closely related to the uplink noise rise is the relative uplink load of the system. In $[1,2,5,6,7]$ the uplink load, $L$ is related to the uplink noise rise $\Lambda$ by

$$
L=1-\frac{1}{\Lambda} \Leftrightarrow \Lambda=\frac{I}{N}=\frac{1}{1-L}
$$

Clearly, $L=0$ implies a noise rise of 1 and hence the total uplink interference is simply the background noise, and as $L$ tends to one, the uplink interference power tends to infinity. As can be seen in Figure 1, it is not recommended having a working point at a too high load level since at high

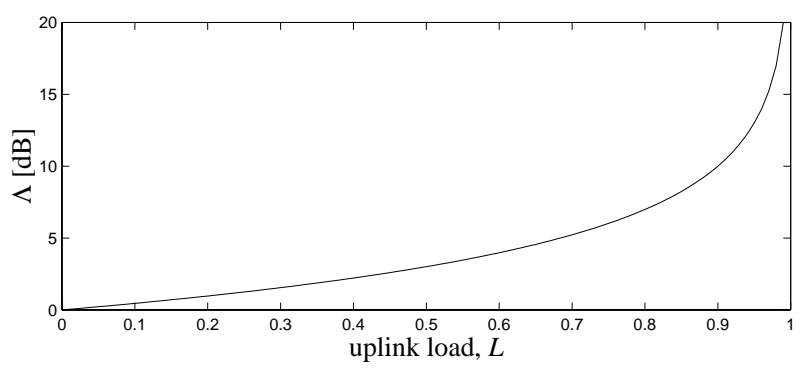

Figure 1: Nonlinear relation between uplink load, $L$ and uplink noise rise.

load levels even a small increase in uplink load corresponds to a substantial interference power increase. Such an increase may, under some circumstances, require unpleasant actions. A noise rise above 4 or $5 \mathrm{~dB}$ is often not considered feasible given power control and maximum transmission power constraints. A noise rise of $4 \mathrm{~dB}$ corresponds to an uplink load of 0.6, which is relatively far from what can be thought of as a theoretical max, somewhere just below one. The relation can for example be useful for an operator wanting to maintain the noise rise of the system below some fixed level. He can then use the simple one-to-one relation and find the corresponding uplink load. This level can be used in various resource management algorithms independently of users' data rate, path loss coefficients and users' location.

An overview of the rest of this paper is as follows. Section 2 presents relevant models and notation. These are utilized in Section 3, where we derive estimates for the uplink noise rise and discuss the strength of the estimates. Section 4 discusses some of the sources of the estimation errors. In Section 5 the estimates are evaluated using simulations. Finally, Section 6 gives some conclusive remarks.

\section{System Model}

Consider a scenario in the uplink of a cellular radio system where we have B base stations (different sectors at the same cell is herein denoted as different base stations) and $\mathrm{M}$ active mobiles. Denote the transmission power of user $i$ with $p_{i}(t), i \in\{1,2, \ldots, M\}$. The signal power used by user $i$ is attenuated by a factor $g_{i, j}(t)(<1)$ before arriving at base 
station $j, j \in\{1,2, \ldots, B\}$. The received carrier power of user $i$ at base station $j$ is then simply $C_{i, j}(t)=p_{i}(t) g_{i, j}(t)$. This signal is added to the other users' signals and background noise in the base station to form the total interference power (total received power):

$$
I_{j}^{t o t}=N_{j}+\sum_{i=1}^{M} p_{i}(t) g_{i, j}(t)
$$

Assume that mobile $i$ is connected solely to base station $j_{i}$. The perceived quality is related to the carrier-to-interferenceratio $(\mathrm{CIR})$ defined by $\gamma_{i}(t)=C_{i, j_{i}}(t) /\left(I_{j_{i}}^{t o t}(t)-C_{i, j_{i}}(t)\right)$. Power control in WCDMA aims at maintaining $\gamma_{i}(t)$ equal to a user dependent target $C I R, \gamma_{i}^{g t}(t)$ [8]. These targets are continuously reconsidered by radio resource management algorithms. For presentational ease we will from here on use the carrier-to-total-interference-ratio (CTIR), $\beta_{i}(t)=$ $C_{i, j_{i}}(t) / I_{j_{i}}^{t o t}(t)$ instead of $\gamma_{i}(t)^{1}$. In the system considered here, a mobile may be connected to several base stations at different physical sites (soft handover) or to several base stations at the same site (softer handover). Utilizing maximum ratio combining of the received signals in softer handover yields:

$$
\beta_{i}(t)=\sum_{k \in K_{i}} \frac{g_{i, k}(t) p_{i}(t)}{I_{k}^{t o t}(t)}
$$

where $K_{i}$ is the set of base stations user $i$ is connected to. Conversely, in the case of soft handover selection combining is used and consequently the signal with the best quality measure is chosen, i.e.

$$
\beta_{i}(t)=\max _{k \in K_{i}} \frac{g_{i, k}(t) p_{i}(t)}{I_{k}^{t o t}(t)}
$$

Note that both soft and softer handover are used concurrently in a true system as well as in the simulator. Maximum in (4) will be taken over the results from the local softer handover combinings if only a subset of the active links are in softer handover.

\section{Uplink Load Estimates}

In this section we derive estimates for the uplink load and state some fundamental requirements. All the estimates use only data readily available in a WCDMA system. Throughout this section the time index $t$ will be suppressed for notational ease when not needed.

\subsection{Approximations and Estimates}

In order to derive the estimates a number of assumptions have to be made. First, assume that the expression for $\beta_{i}$ found in (3) when in softer handover is approximately true for soft handover as well. The assumption allows us to write

$$
\beta_{i}=p_{i} \sum_{k \in K_{i}} \frac{g_{i, k}}{I_{k}^{t o t}} \Leftrightarrow p_{i}=\frac{\beta_{i}}{\sum_{k \in K_{i}} \frac{g_{i, k}}{I_{k}^{o t}}}
$$

\footnotetext{
${ }^{1}$ Note the relation $\beta_{i}=\gamma_{i} /\left(1+\gamma_{i}\right)$.
}

Inserting the above expression for $p_{i}$ into (2) and assuming perfect power control $\left(\beta_{i}=\beta_{i}^{\text {tgt }}\right)$ yield

$$
I_{j}^{t o t}=N_{j}+\sum_{i=1}^{M} g_{i, j} p_{i}=N_{j}+\sum_{i=1}^{M} g_{i, j} \frac{\beta_{i}^{t g t}}{\sum_{k \in K_{i}} \frac{g_{i, k}}{I_{k}^{t o t}}}
$$

In case of soft handover, we get

$$
I_{j}^{t o t}=N_{j}+\sum_{i=1}^{M} g_{i, j} p_{i}=N_{j}+\sum_{i=1}^{M} g_{i, j} \frac{\beta_{i}^{\text {tgt }}}{\max _{k \in K_{i}} \frac{g_{i, k}}{I_{k}^{t o t}}}
$$

At this stage there are two different ways of proceeding in order to solve for $I_{j}^{t o t}$.

- Assume that $I_{k}^{\text {tot }}$ approximately equals $I_{j}^{\text {tot }}$, i.e. we assume the uplink interference power to be approximately the same in all neighboring cells. With this approximation, (6) may be rearranged into

$$
I_{j}^{t o t}=\frac{N_{j}}{1-\sum_{i=1}^{M} \beta_{i}^{t g t} \frac{g_{i, j}}{\sum_{k \in K_{i}} g_{i, k}}}
$$

Comparing this expression to (1), the sum over the users may be identified as an estimate of the uplink load in cell $j, L_{j}$. Hence, estimates of the uplink load and noise rise are

$$
\hat{L}_{j}^{1}=\sum_{i=1}^{M} \beta_{i}^{t g t} \frac{g_{i, j}}{\sum_{k \in K_{i}} g_{i, k}} \Leftrightarrow \hat{\Lambda}_{j}^{1}=\frac{1}{1-\hat{L}_{j}^{1}}
$$

- Assuming $N_{j}=N_{k}$, dividing equation (6) by $N_{j}$ and substituting $\frac{I_{j}^{\text {tot }}}{N_{j}}$ with $\Lambda_{j}$ yield

$$
\Lambda_{j}(t)=1+\sum_{i=1}^{M} \beta_{i}^{t g t}(t) \frac{g_{i, j}(t)}{\sum_{k \in K_{i}} \frac{g_{i, k}(t)}{\Lambda_{k}(t)}}
$$

By assuming $\Lambda_{k}(t)$ to approximately equal $\Lambda_{k}(t-1)$ this too becomes a practically tractable estimate. (the " 2 " indicates the second estimate)

$$
\hat{\Lambda}_{j}^{2}(t)=1+\sum_{i=1}^{M} \beta_{i}^{t g t}(t) \frac{g_{i, j}(t)}{\sum_{k \in K_{i}} \frac{g_{i, k}(t)}{\hat{\Lambda}_{k}^{2}(t-1)}}
$$

According to equation (1) yet another estimate of $L$ can be found from (11):

$$
\hat{L}_{j}^{2}=1-\frac{1}{\hat{\Lambda}_{j}^{2}}
$$

If we, instead of assuming softer handover, assume soft handover is used everywhere we get $\beta_{i}$ according to (4) instead of (3) and hence the sum over $k$ in (5) will be substituted with taking maximum. The corresponding estimates would then be

$$
\hat{L}_{j}^{3}=\sum_{i=1}^{M} \beta_{i}^{t g t} \frac{g_{i, j}}{\max _{k \in K_{i}} g_{i, k}} \Leftrightarrow \hat{\Lambda}_{j}^{3}=\frac{1}{1-\hat{L}_{j}^{3}}
$$


and

$$
\begin{gathered}
\hat{\Lambda}_{j}^{4}(t)=1+\sum_{i=1}^{M} \beta_{i}^{t g t} \frac{g_{i, j}}{\max _{k \in K_{i}} \frac{g_{i, k}}{\hat{\Lambda}_{k}(t-1)}} \\
\hat{L}_{j}^{4}=1-\frac{1}{\hat{\Lambda}_{j}^{4}}
\end{gathered}
$$

\section{Sources of Estimation Error}

A number of different effects and approximations degrade the performance of the load estimate. These include

- the soft(er) handover assumption

- imperfect power control

- the assumption regarding relation between the uplink interference in all cells, alternatively the background noise and the dynamics of the uplink interference

- path gain measurement errors according to [9] and sparsely sampled path gain measurement reports

- the assumption that unknown path gains are equal to zero

- TX rise (see below)

The effects on the estimation error are explored in Section 5 .

\subsection{TX Rise}

In this section we define and discuss $T X$ rise. For simplicity, assume that each user is allowed to connect to no more than one base station at a time in this section. TX rise is the result of the fast power control adjusting each user's transmission power to compensate for fast fading dips in the path gain between mobile and connected base station. The mobile uses a high power during deep fades. This may yield a considerable interference addition to neighboring cells since a dip to the connected base station does not always imply an, as deep, dip to the neighboring base stations.

As an example, we consider mobile 1, which is connected to base station 2 , and study its interference contribution to base station 3. Due to power control, each user's transmission power is approximately inversely proportional to the momentarily path gain between the user and the controlling base station, i.e.,

$$
p_{1} \propto \frac{1}{g_{1,2}}
$$

Base station 3 will receive the signal $p_{1} g_{1,3}$ which is proportional to the ratio between the momentarily path gains, i.e.,

$$
C_{1,3} \propto \frac{g_{1,3}}{g_{1,2}}
$$

The path gain reports provided by the users do not include fast fading (the actual reported value is low pass filtered; $\left.F\left\{g_{i, j}\right\}\right)$. Since the estimates, unlike fast power control, use users' path gain reports there will be a part of the uplink interference power that will not be counted for in the estimates. Essentially, we can observe $\frac{F\left\{g_{1,3}\right\}}{F\left\{g_{1,2}\right\}}$ but not $\frac{g_{1,3}}{g_{1,2}}$, which is related to the actual interference contribution in base station 3. In the top graph of Figure 2 we have plotted the ratio of low-pass filtered versions of the path gain measurements (solid). Of course this will not detect the high peaks in the ratio of the momentarily path gains (dashed). Perhaps momentarily sharp peaks are tolerable by base station 3. A better model could therefore be to consider the low-pass filtered load contribution which is essentially proportional to $F\left\{\frac{g_{1,3}}{g_{1,2}}\right\}$. However, as indicated by the bottom graph in Figure 2, the load contribution is still underestimated. In fact, this exemplifies that

$$
\frac{E\left\{g_{i, j}\right\}}{E\left\{g_{i, k}\right\}} \neq E\left\{\frac{g_{i, j}}{g_{i, k}}\right\}
$$

The solid line in the bottom graph of Figure 2 thus repre-
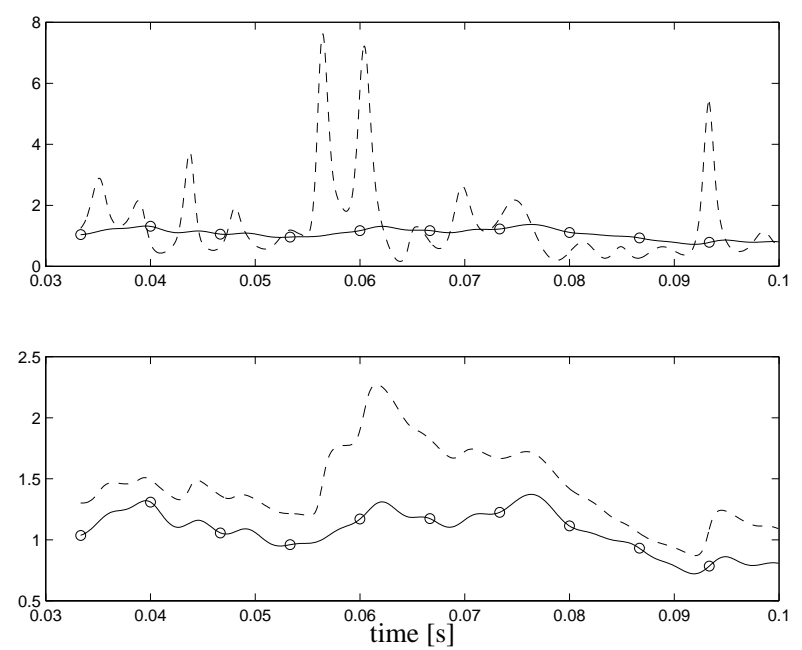

Figure 2: top: Ratio between filtered measurements (solid) and momentary $\frac{g_{1,3}}{g_{1,2}}$ (dashed) bottom: as above but comparing to a filtered ratio, $F\left\{\frac{g_{1,3}}{g_{1,2}}\right\}$

sents the quantity used in our estimates of the average noise rise while the dashed line represents the quantity that corresponds to user $i$ 's actual contribution to the average uplink noise rise in base station $j$.

\section{Simulations}

Simulations have been used to investigate the error of the different estimates at different load levels. Recall the discussion in the introduction, where we pointed out the oneto-one relation between uplink load and noise rise. It is therefore irrelevant if we discuss performance in terms of load or noise rise. We will choose the one which best 
shows the interesting facts. To maintain simulation times to a moderate level, we have used two types of streaming users; $64 \mathrm{kbps}$ and $192 \mathrm{kbps}$. The simulated environment is a suburban situation with 21 base stations, slow moving users $(5 \mathrm{~km} / \mathrm{h})$, path loss coefficient $\alpha=3.5$, shadow fading $\left(d_{0}=100 \mathrm{~m}, \sigma=6 \mathrm{~dB}\right)$, fast fading and RAKE receivers. We have used uniformly distributed users throughout the simulations. Measurements of path gains are subject to measurement errors according to [9], i.e. a $90 \%$ confidence interval of $1.5 \mathrm{~dB}$. To keep signaling to a minimum only path gain measurement to the five strongest base stations are reported by each user. In [10] it is shown that $\hat{L}^{1}$ and $\hat{L}^{2}$ are practically independent of the type of path gain reporting, periodic or event-based, and therefore only event-based reports are considered. To isolate the sources of estimation error considered here, the users have no upper transmission power limit.

\subsection{Single Service}

Figure 3 shows the performance of the four different estimates when using $64 \mathrm{kbps}$ streaming users. As each user has no more than one link, assuming soft or softer handover does not make any difference in this scenario. The major gap between the lines is due to TX rise, since fast fading is not simulated in the runs corresponding to the dashed lines. From this figure we can conclude that TX rise does give a considerable contribution to the total error. Also, all four estimates produce an underestimate of the uplink noise rise, mainly due to that measurements of all path gains are not available or slightly inaccurate. In Figure 4 a more realis-

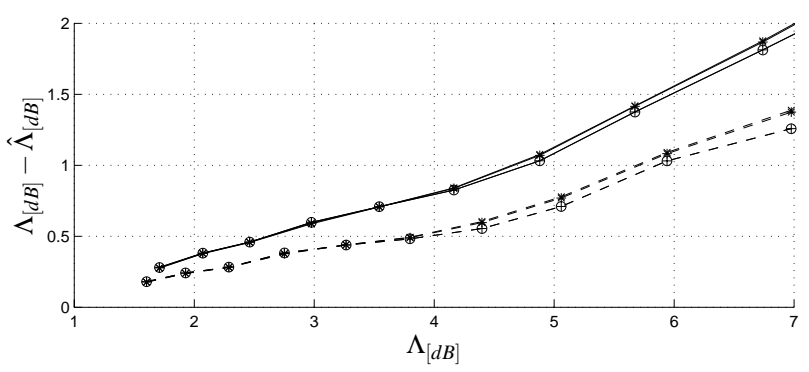

Figure 3: Noise rise estimate error with $64 \mathrm{kbps}$ users. One link per user. Dashed: No fast fading. $\hat{L}^{1}$ : 'o', $\hat{L}^{2}$ 'x', $\hat{L}^{3}{ }^{\prime}{ }^{+}{ }^{\prime}, \hat{L}^{4}:{ }^{*}{ }^{\prime}$

tic scenario is shown where each user is allowed up to three handover links at a time. First of all, assuming softer handover $\left(\hat{L}^{1}\right.$ and $\left.\hat{L}^{2}\right)$ gives larger average error than assuming soft handover $\left(\hat{L}^{3}\right.$ and $\left.\hat{L}^{4}\right)$. Under these circumstances, i.e., almost equal load in all cells, the errors from all four estimates are low in average and has comfortibly small standard deviation. Assuming $I_{k}^{\text {tot }}(t)=I_{k}^{\text {tot }}(t-1)$ (i.e., $\hat{L}^{2}$ and $\hat{L}^{4}$ ) provides estimates with higher average error but with lower standard deviation. In these estimates, we use the previous estimate to estimate each user's contribution to the noise rise. By looking at equation (11), which is repeated below, we realize that underestimating the noise rise at time
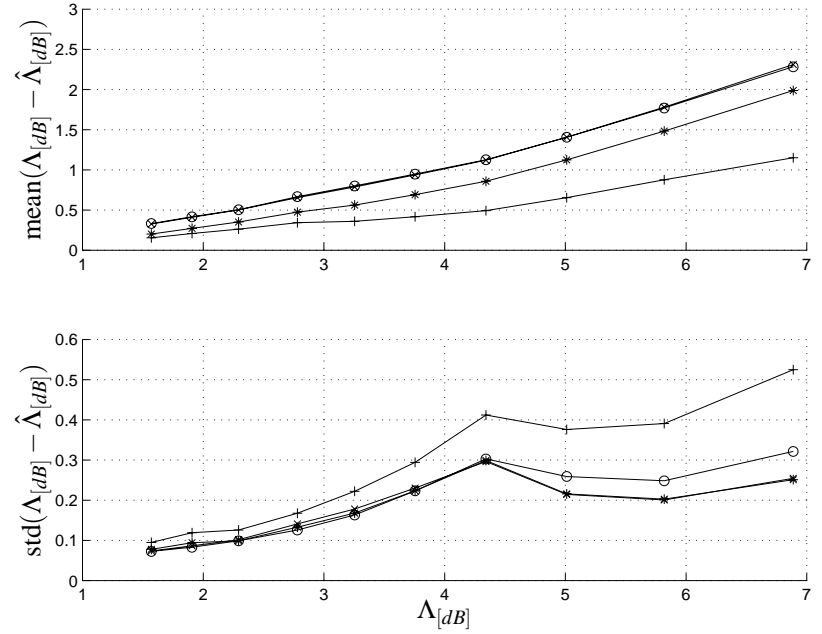

Figure 4: Noise rise estimate error with 64 kbps users. Up to three links per user. Upper: mean. Lower: standard deviation. $\hat{L}^{1}$ : 'o', $\hat{L}^{2}{ }^{\prime}{ }^{\prime}, \hat{L}^{3}:^{\prime}{ }^{+}{ }^{\prime}, \hat{L}^{4}$ : ' ${ }^{*}$ '

$t-1$ results in underestimating every term in the sum that constitutes the noise rise at time $t$.

$$
\hat{\Lambda}_{j}(t)=1+\sum_{i=1}^{M} \beta_{i}^{t g t} \frac{g_{i, j}}{\sum_{k \in K_{i}} \frac{g_{i, k}}{\hat{\Lambda}_{k}(t-1)}}
$$

The lowest line in the upper graph of Figure 4 represents $\hat{L}^{3}$. We can see that it estimates the noise rise with an error less than $1 \mathrm{~dB}$ on average for as high true noise rise as $6 \mathrm{~dB}$. The slight increase in error for $\hat{L}^{3}$ as the noise rise increases, may be explained by looking in the load domain. As mentioned in the introduction, even a small difference in load gives a substantial increase in noise rise at high load levels. This also means that a small error in the uplink load estimate results in a large error in the noise rise estimate when operating at high load levels. In Figure 5 estimated load is plotted versus true load computed according to equation (1) where $\Lambda$ is given by the simulator. In this domain we see no dramatic increase in estimation error as the load of the system increases. All the lines in the figure are quite straight but do not have the correct incline. This can best be explained by studying the left half of equation (13). Ideally, the estimate is a sum over all the users in the network. The fact that the line representing $\hat{L}^{3}$ in Figure 5 does not have the correct incline thus corresponds to that each user's contribution is underestimated. The behavior is more visible when looking at the other estimates, especially $\hat{L}^{1}$ and $\hat{L}^{2}$. Also $\hat{L}^{2}$ and $\hat{L}^{4}$ have a sum over, ideally, all the users, shown in (11), and hence the same reasoning applies. To summarize, there are two reasons why the lines in Figure 4 deviates from a straight line for high load levels:

- even a minor error at high loads produces a nonnegligible error in the noise rise

- the sum representing the load estimate has a larger 
error due to many users, all with a minor underestimated contribution.

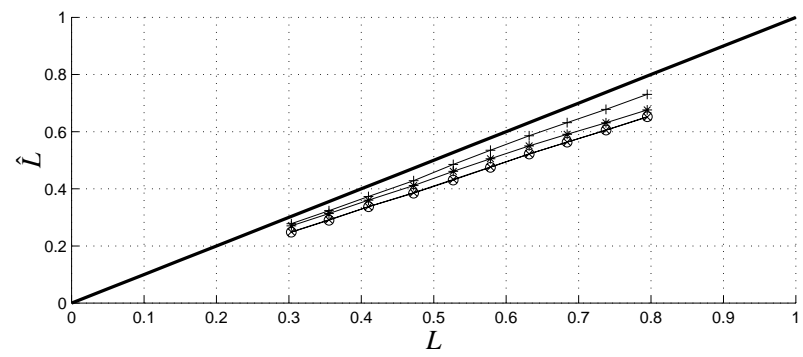

Figure 5: Uplink load estimate error when using $64 \mathrm{kbps}$ users. $\hat{L}^{1}$ : ' o', $\hat{L}^{2}{ }^{\prime} \mathrm{x}$ ', $\hat{L}^{3}$ : ' + ', $\hat{L}^{4}$ : '*'

\subsection{Multiple Services}

In this section we will illustrate the estimates' insensitivity to users' data rate, represented by $\beta_{i}^{\text {tgt }}$. To not make the figure too cluttered, we have chosen to exemplify with $\hat{L}^{3}$ and $\hat{L}^{4}$. The load estimates with two different types of users are depicted in Figure 6. Both estimates are practically independent of the users' data rate. This is a great benefit compared to hard capacity, where each service has to be translated into a number related to the load contribution.

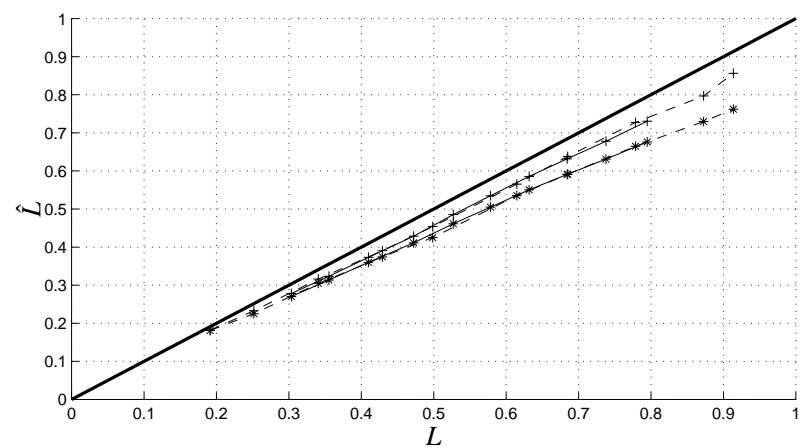

Figure 6: Uplink load estimate error when using $64 \mathrm{kbps}$

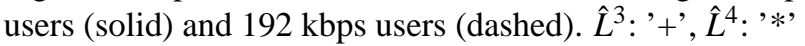

\section{Conclusions}

Uplink noise rise is considered to be a relevant uplink load measure in WCDMA, but it is difficult to measure accurately in practice. In this work, we have proposed and evaluated four different estimates of the uplink noise rise of a WCDMA system. The estimates have different assumptions regarding soft handover and interference power dynamics and correlation between cells. We have shown that, given readily available data, the estimates provide good average accuracy even for high interference levels. The estimate assuming soft handover and that the interference power is approximately constant over a time period of ten milliseconds provides a stable estimate which has low average error in all scenarios simulated, below $1 \mathrm{~dB}$ for moderate interference levels. Furthermore, all estimates inherently handle multi-service scenarios well. Due to the low variation in the single estimate errors, a rather simple correction algorithm should provide even better estimates. The above mentioned properties of the estimates make them well suited for monitoring of the current radio network load as well as for radio resource management.

\section{References}

[1] H. Holma and J. Laakso. Uplink admission control and soft capacity with MUD in CDMA. In Proc. IEEE Vehicular Technology Conference, Amsterdam, the Netherlands, Sep 1999.

[2] C. Y. Huang and Yates R. D. Call admission in power controlled CDMA systems. In Proc. IEEE Vehicular Technology Conference, Atlanta, GA, USA, May 1996.

[3] Y. Ishikawa and N. Umeda. Capacity design and performance of call admission control in cellular CDMA systems. IEEE Journal on Selected Areas in Communications, 15(8), 1997.

[4] E. Geijer Lundin. Uplink admission control based on estimated interference in WCDMA systems. Master Thesis LiTH-ISY-EX-3123, Dept. of Electrical Engineering, Linköpings universitet, Sweden, 2001.

[5] N. Wiberg and A. Gioia. Uplink packet access control in WCDMA. In Proc. IEEE Vehicular Technology Conference, Tokyo, Japan, May 2000.

[6] J. Shapira. Microcell engineering in CDMA cellular networks. IEEE Transactions on Vehicular Technology, 43(4), 1994.

[7] W. Rave, T. Köhler, J. Voigt, and G. Fettweis. Evaluation of load control strategies in an UTRA/FDD network. In Proc. IEEE Vehicular Technology Conference, Rhodes, Greece, May 2001.

[8] F. Gunnarsson. Power Control in Cellular Radio System: Analysis, Design and Estimation. $\mathrm{PhD}$ thesis, Linköpings universitet, Linköping, Sweden, April 2000 .

[9] 3GPP Technical Specification Group Radio Access Network. Requirements for support of radio resource management. Technical report 3G TS 25.133, 2000.

[10] F. Gunnarsson, E. Geijer Lundin, G. Bark, N. Wiberg. Uplink admission control in WCDMA based on relative load estimates. Proc. International Conference on Communications, New York, April 2002. 